\title{
Tildrakizumab in the treatment of psoriasis - literature review
}

\section{Katarzyna Banaszczyk}

Faculty of Medicine, Ludwik Rydygier Collegium Medicum in Bydgoszcz, Nicolaus Copernicus University in Torun, Bydgoszcz, Poland

\begin{abstract}
Psoriasis is a chronic, inflammatory disease. The incidence of its occurrence in developed countries is $1-4 \%$. The aim of this study is to present the current state of knowledge and characterize the antibody tildrakizumab, which in March 2018 was approved by the FDA agency for use in adult patients with moderate to severe plaque psoriasis. The work presents the mechanism of action of tildrakizumab and the way it affects the immunological pathways associated with the pathogenesis of psoriasis. Recent studies indicate that interleukin 23 and its p19 subunit are a key target in the effective treatment of psoriasis. The paper also presents the latest clinical trials that present the safety profile and efficacy of tildrakizumab in the treatment of psoriasis. According to clinical trials, tildrakizumab is an effective and safe drug for use in adult patients in the treatment of moderate to severe psoriasis.
\end{abstract}

Key words: psoriasis, antibodies, interleukins, tildrakizumab.

\section{Introduction}

Psoriasis is a chronic inflammatory disease that affects many body systems. In the majority of cases, the lesions concern skin and joints [1]. In developed countries, the incidence of psoriasis is estimated at about $1-4 \%$ [2]. The most common form of psoriasis is plaque psoriasis, which is characterized by the presence of psoriatic plaques, most commonly found on the scalp and the upright part of the knees and elbows. A disease often associated with psoriasis is psoriatic arthritis. Psoriatic arthritis affects $5 \%$ to $42 \%$ of patients with psoriasis. Interestingly, it can overtake skin symptoms in up to $15 \%$ of patients [3]. It is worth noting that psoriasis is associated with the risk of coexisting diseases such as heart disease, including ischemic heart disease, metabolic syndrome, depression, diabetes, hypertension and inflammatory bowel diseases $[4,5]$.

Due to possible co-morbidities, decreased quality of life and sometimes also disability, this disease is still a challenge for modern medicine [6]. Studies show that patients with psoriasis are characterized by increased mortality compared to healthy people [7].
Research is continuing to explain the exact etiopathogenesis of the disease, and new drugs are being introduced all the time to better control the disease and increase the quality of life of those struggling with psoriasis.

In March 2018, the monoclonal antibody tildrakizumab was approved by the FDA for use in adult patients with moderate to severe plaque psoriasis [8]. The aim of this work is to collect up-to-date information on tildrakizumab and present the latest clinical trials conducted with tildrakizumab.

\section{The mechanism of action of tildrakizumab}

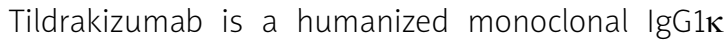
antibody. This antibody selectively binds to the p19 subunit, thereby inhibiting the interaction of IL-23 with its receptor, and thus inhibits the release of $\mathrm{IL}-23$ mediated proinflammatory cytokines [9]. Recent studies show that the IL-23/IL-17 axis plays a key role in the etiopathogenesis of psoriasis [10]. IL-23 is involved in stimulation, and what is more, it also affects the functioning of Th-17 lymphocytes, which play an important role in the pathogenesis of psoriasis [11].

\section{Address for correspondence:}

Katarzyna Banaszczyk, Faculty of Medicine, Ludwik Rydygier Collegium Medicum in Bydgoszcz, Nicolaus Copernicus University in Toruń, 13 Jagiellońska St., 85-067 Bydgoszcz, Poland, e-mail: kbanaszczyk570@gmail.com

Submitted: 20.06.2019; Accepted: 2.08.2019 
The development of the autoimmune response and inflammatory response is associated with IL-23. As results from the conducted research, the lack of IL-23 is associated with the arrest of Th17 cell development at the early stage of activation, and thus with a decrease in the level of proinflammatory IL-17 produced by stimulated Th17 lymphocytes. The lack of IL-23 is also associated with a smaller pool of Th17 lymphocytes located in the lymph nodes [12].

Interleukin 23 is a heterodimeric cytokine consisting of the p19 and p40 subunits [13]. The IL-23 p40 subunit is shared with IL-12. The p40 subunit is the target of another biological drug for psoriasis - ustekinumab. Recent clinical trials suggest that a drug targeting exclusively IL-23 and the p19 subunit may result in a more favorable safety profile compared to targeting both IL-23 and IL-12 [14]. Interleukin 12, as shown by studies, may be of beneficial importance for the organism and participate in the protection of the organism against the action of intracellular pathogens, and what is more, it also probably participates in immune surveillance in the development of cancer [15].

It is also worth noting that inhibition of the p40 subunit is also associated with the inhibition of IL-39, which is still a poorly studied factor, and it is not known whether it performs beneficial functions in the human body [16].

The above information suggests that selective inhibition of p19 subunit activity is safer, among other things due to still insufficient knowledge about the functioning of immunological pathways and the functions of specific interleukins in the human body.

\section{Clinical trials with tildrakizumab in the treatment of plaque psoriasis}

Kopp et al. [17] carried out a three-part, multi-dose, randomized and placebo-controlled clinical trial aimed at determining the safety, tolerability and pharmacokinetics of tildrakizumab, when administered intravenously to patients with moderate to severe plaque psoriasis. The second target of this clinical trial was to evaluate the effectiveness of tildrakizumab in treating this disease.

77 people participated in the study, aged from 22 to 65 years. The first part of this clinical trial was to administer the antibody to patients at certain doses: $0.1 \mathrm{mg} / \mathrm{kg}$ body weight $(n=3), 0.5 \mathrm{mg} / \mathrm{kg}(n=3)$, $3 \mathrm{mg} / \mathrm{kg}(n=6)$ or $10 \mathrm{mg} / \mathrm{kg}(n=6)$ tildrakizumab or placebo $(n=6)$ successively at 0,8 and 12 weeks. Patients were observed for 196 days after the first dose. The second part of the study, which aimed to determine the efficacy of tildrakizumab, consisted of administer- ing higher doses of antibodies to patients - respectively, 40 subjects received $3 \mathrm{mg} / \mathrm{kg}(n=15)$ or $10 \mathrm{mg} / \mathrm{kg}$ $(n=14)$ tildrakizumab or a placebo $(n=11)$; medicines were given at 0, 4 and 8 weeks. The third part of this clinical trial evaluated the efficacy of lower doses of intravenous tildrakizumab. Twelve patients were randomly divided into three groups, at a ratio of $2: 1: 1$. The following doses were given: $0.05 \mathrm{mg}$ kg tildrakizumab $(n=6)$, $0.1 \mathrm{~kg} / \mathrm{mg}$ tildrakizumab $(n=3)$ or placebo $(n=3)$ at weekdays 0,8 and 12 . The primary endpoint of this study was the percentage change in Psoriasis Area and Severity Index (PASI) relative to baseline after 4 or 8 weeks of treatment.

Regarding the results of this clinical trial, the efficacy was unquestionably dependent on the dose of the antibody administered. All patients receiving tildrakizumab in a dose of 3 or $10 \mathrm{mg} / \mathrm{kg}$ achieved a PASI 75 (until 196 day of the study), and many of them had a PASI 90 score; moreover, they maintained at least PASI 50 at week 44 of the study (day 308, 36 weeks after the last dose). Regarding the safety profile, tildrakizumab administered intravenously was generally well tolerated in all doses evaluated. In the second part of this study, 10 of 15 patients in the $3 \mathrm{mg} / \mathrm{kg}$ group and 13 of $14 \mathrm{pa}$ tients receiving $10 \mathrm{mg} / \mathrm{kg}$ achieved PASI 75 until day 112 of the study. The maximum assessed dose was $10 \mathrm{mg} / \mathrm{kg}$. The most common side effects are: headache (tildrakizumab group: 11/57, placebo group: 3/20), upper respiratory tract infection (11/57 and $3 / 20$ respectively), nasopharyngitis (10/57 and $2 / 20$ respectively), and cough (9/57 and 3/20) [17].

This study showed that tildrakizumab provides a significant clinical improvement in patients with moderate to severe psoriasis, as demonstrated by improved PASI scores as well as skin histological samples that were also evaluated in this study.

Papp et al. [18] conducted a randomized, doubleblind, placebo-controlled clinical trial consisting of subcutaneous administration of tildrakizumab to adult patients with moderate to severe plaque psoriasis. The study was conducted in 64 centers in the United States, Canada, Europe and Japan. The study was completed by 266 patients who were subjects with moderate or severe plaque psoriasis (PASI $\geq 12$, BSA $\geq 10$, moderate, severe or severe Physician's Global Assessment - PGA), who had been suffering for at least 6 months. Patients were randomly assigned to receive subcutaneous tildrakizumab at doses of $5 \mathrm{mg}, 25 \mathrm{mg}, 100 \mathrm{mg}, 200 \mathrm{mg}$ or placebo. The intervention took place on week 0 and 4 , then every 12 weeks until week 52. Patients were followed for 72 weeks. The primary endpoint was to achieve the PASI 75 result at week 16 of the study. 
At week 16 of the study, PASI 75 was obtained in $33 \%, 64 \%, 66 \%$ and $74 \%$ of patients at a dose of $5 \mathrm{mg}$ $(n=42), 25 \mathrm{mg}(n=90), 100 \mathrm{mg}(n=89)$, and $200 \mathrm{mg}$ $(n=86)$ of tildrakizumab compared to $4.4 \%$ achieved with placebo. The result of PASI 75 was maintained at week 52 in $97 \%$ of patients who continued the $100 \mathrm{mg}$ dose $(n=30 / 31)$ or $200 \mathrm{mg}(n=29 / 32)$. From the above clinical trial, it appears that tildrakizumab achieved better treatment efficacy than placebo, which was maintained for 52 weeks of treatment and for 20 weeks after discontinuation of antibody therapy. Tildrakizumab was a safe and well-tolerated drug [18].

\section{What results from the clinical trials reSURFACE 1 and reSURFACE 2?}

Reich et al. [19] carried out two parallel, three-part clinical trials. These were double-blind, randomized and placebo-controlled studies. The aim of this study was to determine whether tildrakizumab is better than placebo and etanercept in the treatment of chronic plaque psoriasis. The first study, called reSURFACE 1, was conducted at 118 locations in Australia, Canada, Japan, the United Kingdom and the United States, and lasted from December 10, 2012 to October 28, 2015. A parallel reSURFACE 2 study was carried out in 132 places in Europe, Israel and the USA and lasted from February 12, 2013 to September 28, 2015.

The study involved patients over 18 years of age who suffered from moderate to severe plaque psoriasis. They fulfilled the following conditions for assessing the severity of psoriasis: body surface area involvement $\geq 10 \%$, PGA score $\geq 3$, and PASI score $\geq 12$. Patients were divided into two groups.

In the reSURFACE 1 study, 308 patients received tildrakizumab at a dose of $200 \mathrm{mg}, 309$ patients received this drug at a dose of $100 \mathrm{mg}$, and another subgroup consisted of 155 patients who received a placebo. In total, 772 patients participated in the reSURFACE 1 study. As can be seen from the above information, patients were divided in a $2: 2: 1$ ratio. In a parallel reSURFACE 2 study, 314 patients received tildrakizumab 200 mg, 307 patients received tildrakizumab 100 mg, 156 patients received placebo, and 313 etanercept. As can be seen from the above data, patients were divided in a $2: 2: 1: 2$ ratio. A total of 1,090 patients participated in the reSURFACE 2 study. It is worth noting that randomization was conducted in terms of body weight ( $\leq 90 \mathrm{~kg}$ or $>90 \mathrm{~kg}$ ) and previous exposure to biological treatment. Tildrakizumab was administered subcutaneously to patients in week 0 and 4 during the first part of the study and the 16th week of the study during the second part of the study. The primary endpoints were the proportions of patients achieving a PASI 75 and PGA response (score 0 or 1 with a reduction $\geq 2$ degrees from baseline) at week 12 of the study.

Regarding the reSURFACE 1 study, the following results were obtained. At week 12 of the study, 192 patients (which was 62\%) in the tildrakizumab $200 \mathrm{mg}$ group and 197 patients (64\%) in the $100 \mathrm{mg}$ tildrakizumab group achieved a PASI 75 score, compared to 9 patients (which was 6\%) in the placebo group. Regarding the second primary endpoint, 82 patients (59\% respectively) in the tildrakizumab 200 mg group and 179 patients (representing 58\%) in the $100 \mathrm{mg}$ tildrakizumab group achieved the desired PGA response, compared to 11 patients (which was 7\%) in the placebo group. The parallel study, reSURFACE 2, brought the following results. At week 12 of this study, 206 patients (66\%) in the tildrakizumab $200 \mathrm{mg}$ group and 188 patients (corresponding to 61\%) in the $100 \mathrm{mg}$ tildrakizumab group achieved the primary endpoint of PASI 75, compared to 9 patients (6\%) in the placebo group and 151 patients (48\%) in the etanercept group. Regarding the second primary endpoint, 186 patients (59\%) in the tildrakizumab $200 \mathrm{mg}$ group and 168 patients (59\%) in the $100 \mathrm{mg}$ tildrakizumab group achieved a PGA response, compared to 7 patients (4\%) in the placebo group and 149 patients (which accounted for $48 \%$ ) in the group receiving etanercept. The above study showed that tildrakizumab $200 \mathrm{mg}$ and $100 \mathrm{mg}$ were effective compared to placebo and etanercept in the treatment of patients with moderate to severe chronic psoriasis. What is more, tildrakizumab was well tolerated by patients. The most common ( $\geq 1 \%$ ) side effects associated with the use of tildrakizumab included upper respiratory tract infections, injection site reactions and diarrhea. Adverse reactions that occurred with frequency less than $1 \%$ but greater than $0.1 \%$ were dizziness and pain in the limbs [19].

\section{The safety of tildrakizumab in healthy subjects}

An interesting study on tildrakizumab was carried out by Khalilieh et al. [20]. The aim of this study was to evaluate the safety of tildrakizumab in healthy subjects. Tildrakizumab was administered subcutaneously and intravenously. The first part of the study $(n=29)$ consisted of intravenous administration of tildrakizumab or a placebo. Tildrakizumab and placebo were administered in the following doses and proportions $-0.1 \mathrm{mg} / \mathrm{kg}$ $(n=4) ; 0.5 \mathrm{mg} / \mathrm{kg}(n=4) ; 3 \mathrm{mg} / \mathrm{kg}(n=8) ; 10 \mathrm{mg} / \mathrm{kg}$ $(n=6)$; placebo $(n=7)$. The study was completed by 22 patients. Discontinuation of the study was related to the withdrawal of consent and in one case to becoming pregnant. The second part of the study consisted of 
administering tildrakizumab subcutaneously to healthy subjects $(n=37)$. The first subgroup of this study $(n=18)$ received $50 \mathrm{mg}$ of tildrakizumab subcutaneously $(n=14)$ and placebo $(n=4)$. The second subgroup received subcutaneously $200 \mathrm{mg}$ of tildrakizumab $(n=14)$ and placebo $(n=5)$.

Regarding the results of this study, among subjects in the intravenous group, $83 \%$ of respondents reported at least one adverse event in the course of the study. It was respectively 2 people (50\%) in the group of $0.1 \mathrm{mg} / \mathrm{kg}$, 4 people (100\%) in the $0.5 \mathrm{mg} / \mathrm{kg}$ group, 7 people (88\%) in the $3 \mathrm{mg} / \mathrm{kg}$ group, 6 people $(100 \%)$ in the $10 \mathrm{mg} / \mathrm{kg}$ group and 5 people (71\%) in the placebo group. The most frequent adverse events were upper respiratory tract infections $(n=11)$, headache $(n=8)$, hematomas at the puncture site $(n=4)$, lethargy $(n=3)$, pharyngitis $(n=3)$, dysmenorrhea $(n=2)$, nasopharyngitis $(n=2)$, nausea $(n=2)$, rhinitis $(n=2)$ and seasonal allergy $(n=2)$. A serious adverse event occurred in one patient who received a placebo, i.e. upper airway obstruction and rhinitis surgery, and in 1 patient receiving tildrakizumab $10 \mathrm{mg} / \mathrm{kg}$ - he was hospitalized due to fever caused by chikungunya virus infection.

None of the serious adverse events were combined with the treatment. The most frequent adverse events associated with tildrakizumab were headache $(n=3)$ and upper respiratory tract infection $(n=2)$. There was no increase in the frequency of adverse effects due to the higher dose administered. In the subcutaneous treatment group, 24 subjects (65\%) reported at least one adverse event during the study: 9 subjects (64\%) in the $50 \mathrm{mg}$ maintenance group, 8 (57\%) in the group receiving $200 \mathrm{mg}$ and 7 (78\%) patients in the placebo group. The most common events observed in all treatment groups, including the placebo group, were upper respiratory tract infections $(n=12)$ and headache $(n=10)$. No serious adverse events were observed. Other observed adverse events include acne $(n=2)$, gastroenteritis $(n=2)$, erythema at the injection site $(n=2)$, insomnia $(n=2)$, migraine $(n=2)$, allergic rhinitis $(n=2)$ and rash $(n=2)$. Two patients receiving a $50 \mathrm{mg}$ dose reported three serious adverse events - headache, hypersensitivity to co-administered medicines and viral gastroenteritis - which were considered unrelated to treatment, and one patient in the placebo group reported a serious adverse event, i.e. migraine, which also was considered unrelated to treatment. No association of events not related to the administered dose was noted. There were no serious adverse events in the $200 \mathrm{mg}$ dose group. At the injection site, a mild reaction (slight erythema) was observed in 2 subjects receiving $200 \mathrm{mg}$ tildrakizumab and 2 in the placebo group $[20,21]$.
The study shows that single intravenous administration of tildrakizumab in doses of $0.1 ; 0.5 ; 3$ and $10 \mathrm{mg} / \mathrm{kg}$ and a single subcutaneous administration of this drug at doses of $50 \mathrm{mg}$ and $200 \mathrm{mg}$ are safe and well tolerated in healthy adults.

\section{Tildrakizumab and psoriatic arthritis - recent clinical trials}

Research on the use of tildrakizumab in the treatment of psoriatic arthritis is ongoing. Mease et al. [22] conducted a randomized, double-blind, placebo-controlled clinical trial aimed at determining the efficacy and safety of tildrakizumab in the treatment of patients with active psoriatic arthritis. Patients were divided into five subgroups in a $1: 1: 1: 1: 1$ ratio. Patients received treatment in injections. The first subgroup $(n=78)$ received $200 \mathrm{mg}$ tildrakizumab every 4 weeks, the second $(n=79) 200 \mathrm{mg}$ every 12 weeks, the third ( $n=77) 100$ mg every 12 weeks, the fourth $(n=78) 20 \mathrm{mg}$ every 12 weeks, the fifth group $(n=79)$ received placebo every 4 weeks. Antibody treatment was used until week 24 . The primary endpoint in this study was to achieve a reduction of at least $20 \%$ in the ACR20 criteria at week 24.

At week 24, patients receiving tildrakizumab achieved significantly better PASI scores and a 50\% reduction in the American College of Rheumatology response criteria (ACR50) compared to placebo. Patients who received $200 \mathrm{mg}$ of antibody every 12 weeks in $79.6 \%$ and 50\% achieved PASI 75 and PASI 90, respectively, compared to $16.7 \%$ and $7.1 \%$ in the placebo group. It is worth noting that the response rate improved with the increase in the dose of antibody, but shortening the interval between doses from 12 to 4 weeks did not improve the results in relation to the skin or joints.

Tildrakizumab was safe to use. Severe adverse events were observed in $2.2 \%$ of patients treated with tildrakizumab and $2.5 \%$ of patients receiving placebo. The most common adverse events included: nasopharyngitis (5.4\% - 17/312 in the antibody groups and $6.3 \%-5 / 79$ in the placebo group) and diarrhea (3\% - 4/312 in the antibody groups and 0 cases in the placebo group). There were no undesirable cardiac events, candidiasis, inflammatory bowel diseases or cancers. Importantly, no patient stopped treatment and no deaths were reported [22].

The study shows that tildrakizumab may be an effective drug for patients with psoriatic arthritis, but more clinical trials are needed to assess its efficacy and safety.

\section{Conclusions}

Clinical studies and their results suggest that IL-23 and its p19 subunit are a key target in the effective treatment of moderate to severe plaque psoriasis. There is 
a need for further research to continuously develop psoriasis therapy and achieve the best control of this severe disease, which significantly reduces the quality of life of patients.

Tildrakizumab is a promising drug that, when implemented in clinical practice, may be a good solution for the biological treatment of plaque psoriasis in the moderate or severe stage. It is worth considering the use of this drug in the treatment of patients with psoriasis, when other treatments did not bring the expected results and did not improve the course of the disease.

Furthermore, tildrakizumab has been shown to be effective in the treatment of psoriatic arthritis, which gives hope for better control of this chronic disease in the future. Interleukin 23 and its p19 subunit also play an important role in Crohn's disease, so in the future we can expect clinical trials evaluating the effectiveness of tildrakizumab in treating this disease [22].

The author declares no conflict of interest.

\section{References}

1. Griffiths CE, Barker JN. Pathogenesis and clinical features of psoriasis. Lancet 2007; 370: 263-271.

2. Parisi R, Symmons DP, Griffiths CE, et al. Global epidemiology of psoriasis: a systematic review of incidence and prevalence. J Invest Dermatol 2013; 133: 377-385.

3. Habif TP. Psoriasis and other papulosquamous diseases. In: Clinical Dermatology, Habif TP (ed.), $6^{\text {th }}$ ed. Elsevier, Philadelphia 2016: 263-328.

4. Takeshita J, Grewal S, Langan SM, et al. Psoriasis and comorbid diseases: epidemiology. J Am Acad Dermatol 2017; 76: 377-390.

5. Furue M, Tsuji G, Chiba T, Kadono T. Cardiovascular and Metabolic diseases comorbid with psoriasis: Beyond the skin. Intern Med 2017; 56: 1613-1619.

6. Boehncke WH, Schön MP. Psoriasis. Lancet 2015; 386: 983-994.

7. Masson W, Rossi E, Galimberti ML, et al. Mortality in patients with psoriasis. A retrospective cohort study. Med Clin 2017; 148: 483-488.

8. Tildrakizumab US prescribing information. 2018. https://www. accessdata.fda.gov/drugsatfda_docs/label/2018/761067s000lbl.pdf (accessed: April 21, 2019).

9. European Medicines Agency. IlumetriTM (tildrakizumab): EU summary of product characteristics. 2018.
10. Puig $\mathrm{L}$. The role of IL 23 in the treatment of psoriasis. Expert Rev Clin Immunol 2017; 13: 525-534.

11. Cargill M, Schrodi SJ, Chang M, et al. A large-scale genetic association study confirms IL12B and leads to the identification of IL23R as psoriasis-risk genes. Am J Hum Genet 2007; 80: 273-290.

12. McGeachy MJ, Chen Y, Tato CM, et al. The interleukin 23 receptor is essential for the terminal differentiation of interleukin 17-producing effector T helper cells in vivo. Nat Immunol 2009; 10: 314-324.

13. Lee E, Trepicchio WL, Oestreicher JL, et al. Increased expression of interleukin 23 p19 and p40 in lesional skin of patients with psoriasis vulgaris. J Exp Med 2004; 199: 125-130.

14. Fitch E, Harper E, Skorcheva I, et al. Pathophysiology of psoriasis: recent advances on IL-23 and Th17 cytokines. Curr Rheumatol Rep 2007; 9: 461-467.

15. Hamza T, Barnett JB, Li B. Interleukin 12 a key immunoregulatory cytokine in infection applications. Int J Mol Sci 2010; 11: 789-806.

16. Wang X, Wei Y, Xiao H, et al. A novel IL-23p19/Ebi3 (IL-39) cytokine mediates inflammation in Lupus-like mice. Eur J Immunol 2016; 46: 1343-1350.

17. Kopp T, Riedl E, Bangert C, et al. Clinical improvement in psoriasis with specific targeting of interleukin-23. Nature 2015; 521: 222-226

18. Papp K, Thaçi D, Reich K, et al. Tildrakizumab (MK-3222), an anti-interleukin-23p19 monoclonal antibody, improves psoriasis in a phase $\mathrm{Ilb}$ randomized placebo-controlled trial. $\mathrm{Br}$ J Dermatol 2015; 173: 930-939.

19. Reich K, Papp KA, Blauvelt A, et al. Tildrakizumab versus placebo or etanercept for chronic plaque psoriasis (reSURFACE 1 and reSURFACE 2): results from two randomised controlled, phase 3 trials. Lancet 2017; 390: 276-288.

20. Khalilieh S, Hodsman P, Xu C, et al. Pharmacokinetics of Tildrakizumab (MK-3222), an Anti-IL-23 Monoclonal Antibody, After Intravenous or Subcutaneous Administration in Healthy Subjects. Basic Clin Pharmacol Toxicol 2018; 123: 294-300.

21. Ma C, Jairath V, Khanna R, Feagan BG. Investigational drugs in phase I and phase II clinical trials targeting interleukin 23 (IL-23) for the treatment of Crohn's disease. Expert Opin Investig Drugs 2018; 27: 649-660.

22. Mease PJ, Chohan S, Fructuoso FJG, et al. Randomised, double-blind, placebo-controlled, multiple-dose, Phase 2B study to demonstrate the safety and efficacy of tildrakizumab, a high-affinity anti-interleukin-23p19 monoclonal antibody, in patients with active psoriatic arthritis. EULAR 2019; Abstract LB0002. 